\title{
Is there a role for aortic valve balloon valvuloplasty in the modern era?
}

\section{(Dvonimir Ostojić*, DVlatka Rešković Lukšić, \\ (DMarija Mance, Blanka Glavaš Konja, DMartina Lovrić Benčić, \\ DMaja Strozzi, DJoško Bulum, DJadranka Šeparović Hanževački}

University of Zagreb School of Medicine, University Hospital Centre Zagreb, Zagreb, Croatia
RECEIVED:

February 28, 2019

ACCEPTED:

March 24, 2019

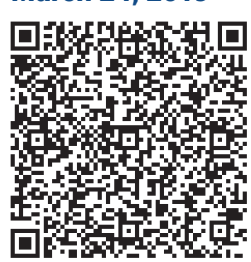

KEYWORDS: aortic stenosis, balloon valvuloplasty, heart failure

CITATION: Cardiol Croat. 2019;14(3-4):95. | https://doi.org/10.15836/ccar2019.95

*ADDRESS FOR CORRESPONDENCE: Zvonimir Ostojić, Klinički bolnički centar Zagreb, Kišpatićeva 12, HR-10000 Zagreb, Croatia. / Phone: +385-91-895-0702 / E-mail: ostojiczvonimir@gmail.com

ORCID: Zvonimir Ostojić, https://orcid.org/0000-0003-1762-9270 • Vlatka Rešković Lukšić, https://orcid.org/0000-0002-4721-3236 Marija Mance, https://orcid.org/0000-0003-1542-2890 • Blanka Glavaš Konja, https://orcid.org/0000-0003-1134-4856 Martina Lovrić Benčić, https://orcid.org/0000-0001-8446-6120 • Maja Strozzi, https://orcid.org/0000-0003-4596-8261 Joško Bulum, https://orcid.org/0000-0002-1482-6503 • Jadranka Šeparović Hanževački, https://orcid.org/0000-0002-3437-6407

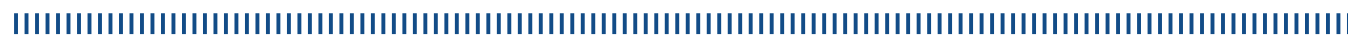

Aim. To investigate early outcomes after balloon aortic valve valvuloplasty (BAV) performed as palliative procedure in patients with decompensated unstable end-stage aortic stenosis (AS).

Patients and Methods. A retrospective observational study was conducted in University Hospital Centre Zagreb. It included all patients who underwent lifesaving BAV, and had no options for surgical or transcatheter aortic valve replacement at the time, between 2015 and 2018. Clinical and echocardiographic characteristics before and after BAV procedure were recorded.

Results. Study included 29 patients, mean age 80.2 (51-92) years, 65.5\% women. Mean logistic Euroscore II was $24.1 \pm 21.1 \%$ and STS score $16.9 \pm 14.5 \%$. Comorbidities included: coronary artery disease $(48.28 \%)$ diabetes mellitus (31.4\%), chronic renal insufficiency (58.6\%), peripheral artery disease (17.2 \%) and atrial fibrillation (55.2\%). All patients were dependent on parenteral diuretics and $24.14 \%$ on parenteral inotropic support. Echocardiographic parameter before and after BAV are presented in table $\mathbf{1}$ There was significant increase in indexed aortic valve area and decrease in pulmonary artery systolic pressure in overall population. There was no significant change in severity of aortic insufficiency. Inhospital mortality was $20.1 \%(\mathrm{~N}=6)$. Periprocedural complications included: 1 (3.4\%) myocardial infarction, 1 (3.4\%) major bleeding, 1 (3.4\%) acute kidney injury and 2 (6.9\%) vascular access complications. All other patients have been successfully weaned of parenteral therapy within 4 days and discharged.

TABLE 1. Echocardiographic parameters before and after aortic valve balloon valvuloplasty.

\begin{tabular}{llll} 
& Before BAV (n=29) & After BAV (N=25) & P value \\
\hline Ejection fraction (\%) & $35.6 \pm 12.3$ & $36.9 \pm 13.8$ & 0.691 \\
\hline E/E' & $25.7 \pm 8.8$ & $25.1 \pm 13.2$ & 0.821 \\
\hline Maximal pressure gradient - $\mathrm{mmHg}$ & $71.1 \pm 42.1$ & $56.8 \pm 31.1$ & 0.134 \\
\hline Mean pressure gradient - $\mathrm{mmHg}$ & $41.7 \pm 24.1$ & $31.1 \pm 14.9$ & 0.055 \\
\hline Indexed aortic valve area & $0.341 \pm 0.008$ & $0.399 \pm 0.12$ & 0.030 \\
\hline Any aortic insufficiency N (\%) & $22(75.9)$ & $21(84)$ & 0.459 \\
\hline More than mild mitral insufficiency N (\%) & $18(62.1)$ & $17(68)$ & 0.649 \\
\hline Pulmonary artery systolic pressure - $\mathrm{mmHg}$ & $64.8 \pm 27.1$ & $46.8 \pm 17.5$ & 0.001 \\
\hline
\end{tabular}

$\mathrm{BAV}=$ balloon aortic valve valvuloplasty

Conclusion. BAV represents palliative treatment option in critically ill hemodynamically unstable patients with end-stage AS, who are not candidates for valve replacement. ${ }^{1}$ BAV provides clinical improvement due to increase AVA and decrease in PAPs, with acceptable mortality and complications rate.

LITERATURE IIIIIIIIIIIIIIIIIIIIIIIIIIIIIIIIIIIIIIIIIIIIIIIIIIIIIIIIIIIIIIIIIIIIIIIIIIIIIIIIIIIIIIIIIIIIIIIIIIIIIII . Pendyala LK, Ben-Dor I, Waksman R. Evolution of percutaneous balloon aortic valvuloplasty in the treatment of patients with aortic stenosis. Minerva Med. 2012 Dec;103(6):415-29. PubMed: https://www.ncbi.nlm.nih.gov/pubmed/23229364 